\title{
TORUS ACTIONS ON COHOMOLOGICAL COMPLEX PROJECTIVE SPACES
}

\author{
KAI WANG
}

\begin{abstract}
In this note, we will compute the Pontrjagin classes of those topological manifolds which have the same rational cohomology algebra as $C P^{n}$ and which admit effective topological $T^{n}$ actions.
\end{abstract}

In [3], Petrie has proved the following:

THEOREM 1. Let $X$ be a closed smooth manifold which has the same homotopy type as $C P^{n}$. If $X$ admits an effective smooth $T^{n}$ action, then $p(X)=$ $\left(1+a^{2}\right)^{n+1}$ where $a \in H^{2}(X ; Z)$ is a generator and $p(X)$ is the total Pontrjagin class of $X$.

It has been shown in [4] that the rational Pontrjagin class can be defined for topological manifolds and euclidean bundles such that the Hirzebruch Index Theorem holds. In this note, we will extend Petrie's result to the topological category in which the $T^{n}$ action on a topological manifold is said to be topological if the fixed sets of any circle subgroups of $T^{n}$ are topological submanifolds. Our main result is the following:

THEOREM 2. Let $X$ be a closed topological manifold such that

$$
H^{*}(X ; Q)=Q[a] /\left(a^{n+1}\right), \quad \operatorname{deg} a=2 .
$$

If $X$ admits an effective topological $T^{n}$ action, then $p(X)=\left(1+a^{2}\right)^{n+1}$.

Proof. We will prove it by induction on $n$. It is obvious that the statement is true for $n=1$. Now we assume that the statement is true for $n=k-1$. Let $X$ be a closed topological manifold such that $H^{*}(X ; Q)=$ $Q[a] /\left(a^{k+1}\right), \operatorname{deg} a=2$, which admits an effective topological $T^{k}$ action. Let $E_{T^{k}} \rightarrow B_{T^{k}}$ be a universal principal $T^{k}$ bundle. We define $X_{T^{k}}$ by $X_{T^{k}}=X$ $\times_{T^{k}} E_{T^{k}}$. It is well known that the following sequence is exact:

$$
0 \rightarrow H^{2}\left(B_{T^{k}} ; Q\right) \rightarrow H^{2}\left(X_{T^{k}} ; Q\right) \rightarrow H^{2}(X ; Q) \rightarrow 0 .
$$

Let $\alpha \in H^{2}\left(X_{T^{k}} ; Q\right)$ be a lifting of $a \in H^{2}(X ; Q)$ and let $g(\alpha)$ be the defining relation of $H^{*}\left(X_{T^{k}} ; Q\right)$ as an algebra over $H^{*}\left(B_{T^{k}} ; Q\right)$. It follows easily from a result of Hsiang [2] that there exist $\omega_{i} \in H^{2}\left(B_{T^{k}} ; Q\right), i=$ $0,1, \ldots, k$, such that $g(\alpha)=\prod_{i=0}^{k}\left(\alpha-\omega_{i}\right)$. Note that we can always choose

Received by the editors August 20, 1976.

AMS (MOS) subject classifications (1970). Primary 57E10, 57E15, 57E25.

Key words and phrases. Pontrjagin classes, defining polynomials.

○ American Mathematical Society 1977 
a lifting so that $\omega_{0}=0$. We will assume from now on that $\omega_{0}=0$. We may consider $\omega_{i}$ as a homomorphism from $T^{k}$ to $S^{1}$. Then $\left(\omega_{1}, \ldots, \omega_{k}\right): T^{k} \rightarrow T^{k}$ is a homomorphism. If the identity component of $\operatorname{ker}\left(\omega_{1}, \ldots, \omega_{k}\right)$ is not trivial, then there is an $S^{1} \subset \operatorname{ker}\left(\omega_{1}, \ldots, \omega_{k}\right)$. It is easy to see that the defining polynomial of $H^{*}\left(X_{S^{1}} ; Q\right)$, where the $S^{1}$ action on $X$ is induced by the inclusion $S^{1} \subset T^{k}$ as an algebra over $H^{*}\left(B_{S^{1}} ; Q\right)$, is given by $h(\beta)=$ $\beta^{k+1}$ where $\beta \in H^{2}\left(X_{S^{1}} ; Q\right)$ is a suitable lifting of $a \in H^{2}(X ; Q)$. It follows from [2] that $S^{1}$ acts trivially on $X$ which contradicts the assumption that the $T^{k}$ action is effective. Hence the identity component of $\operatorname{ker}\left(\omega_{1}, \ldots, \omega_{k}\right)$ is trivial. It follows easily that the identity component of the subgroup $\{t \in$ $\left.T^{k} \mid \omega_{1}(t)=\cdots=\omega_{k}(t)\right\}$ is $S^{1}$. Then the defining polynomial of $H^{*}\left(X_{S^{1}} ; Q\right)$ as an algebra over $H^{*}\left(B_{S^{1}} ; Q\right)$ where the $S^{1}$ action on $X$ is induced by the inclusion $S^{1} \subset T^{k}$ is given by $h(\beta)=\beta(\beta-\omega)^{k}$ for some $\omega \in H^{2}\left(B_{S^{1}} ; Q\right)$ and where $\beta \in H^{2}\left(X_{S^{1}} ; Q\right)$ is a suitable lifting of $a \in H^{2}(X ; Q)$. Again, it follows from [2] that the fixed point set $X^{S^{1}}$ consists of an isolated point and a codimension 2 submanifold $Y$ (note that the action is topological). It is well known that $H^{*}(Y ; Q)=Q[b] /\left(b^{k}\right)$ where $b=i^{*} a, i: \quad Y \rightarrow X$ is the inclusion. It is easy to see that $T^{k} / S^{1} \cong T^{k-1}$ acts effectively and topologically on $Y$. It follows by the induction hypothesis that $p(Y)=\left(1+b^{2}\right)^{k}$. Let $\nu$ be the normal euclidean bundle of $Y$ in $X$. With the same proof of [1, Theorem 4.8.1] we can show that the euler class of $\nu$ is given by $\chi(\nu)=b$. It follows that $p(\nu)=1+b^{2}$. Hence

$$
i^{*} p(X)=p(Y) p(\nu)=\left(1+b^{2}\right)^{k}\left(1+b^{2}\right)=\left(1+b^{2}\right)^{k+1 .}
$$

Since $i^{*} a^{j}=b^{j}$ for $j=1, \ldots, k-1$ and $i^{*} a^{k}=0, p(X)=\left(1+a^{2}\right)^{k+1}+$ $l a^{k}$ for some $l$. However, since $\operatorname{ind}(X)=\operatorname{ind}\left(C P^{k}\right)=k+1$ and the Hirzebruch Index Theorem holds for rational Pontrjagin classes, it follows that $l=0$. This completes the proof.

RemarKs. 1. It is easy to see that any locally smooth action is topological and there are actions on euclidean space whose fixed sets are not locally euclidean at the origin.

2. There are several other generalizations of Petrie's result which will appear in a forthcoming paper [5].

\section{REFERENCES}

1. F. Hirzebruch, Topological methods in algebraic geometry, 3rd ed., Springer-Verlag, Berlin and New York, 1966. MR 34 \#2573.

2. W. Y. Hsiang, Cohomology theory of topological transformation groups, Springer-Verlag, Berlin and New York, 1975.

3. T. Petrie, Torus actions on homotopy complex projective spaces, Invent. Math 20 (1973), 139-146. MR 48 \# 1254.

4. J. A. Schafer, Topological Pontrjagin classes, Comment. Math. Helv. 45 (1970), 315-332. MR 43 \#1199.

5. K. Wang, Torus actions on homotopy complex projective spaces, SUNY at Buffalo, 1976 (preprint).

Department of Mathematics, State University of New York at Buffalo, Amherst, New YoRK 14226 